\title{
Les contre-écritures
}

de Maine de Biran (1766-1824) et de Samuel Taylor Coleridge (1772-1834)

\section{Présentation de l'auteur :}

Titulaire d'un doctorat (Université d'Aston, Birmingham, 2011) sur les conceptions de la volonté de Maine de Biran et Samuel Taylor Coleridge, et ayant enseigné le français, la littérature (de la Renaissance au Nouveau Roman, en passant par les Lumières et le romantisme) I'histoire de France (depuis la révolution), la philosophie et l'histoire des idées (interprétations de la révolution en France, en Grande-Bretagne et en Allemagne, réactions à l'utilitarisme de Jeremy Bentham en France et en Grande-Bretagne victorienne...) à Birmingham, Liverpool, Warwick et aujourd'hui à Londres (University College London), je m'intéresse tout particulièrement à la période qui suit la révolution de 1789 et pendant laquelle l'industrialisation de la France et du Royaume-Uni s'accompagne d'une idéologie fondée sur des prémisses empiristes et à prétention scientifique. L'utilitarisme, comme mode de justification du capitalisme émergent, est au cœur de mes préoccupations.

\section{Résumé}

Dans un monde désenchanté où aucun « lecteur universel » n'attend que nous produisions quoi que ce soit, et où toute validation ne peut être que circonscrite et conditionnelle, il peut être de plus en plus difficile de se convaincre qu'écrire compte. Une conscience aigüe de ce que le climat intellectuel et culturel tournait à un utilitarisme mettant l'accent sur la gratification immédiate, il y a quelque deux siècles de cela, a amené Maine de Biran et Samuel Taylor Coleridge à écrire, en marge de leurs publications plus ou moins bien pensées et reçues, contre le public, au sein de leurs Journal et Notebooks respectifs. Ces écrits, le lieu d'une exploration du moi supposée ne jamais passer le seuil de l'édition, étaient ainsi le lieu d'une résistance à la logique implacable de la recherche de reconnaissance, recherche d'autant plus douloureuse qu'elle était, pour Biran et Coleridge, vouée à demeurer insatiable. Jean-Paul Sartre avance cependant que ce genre de résistance au lectorat, particulièrement dans le contexte du dix-neuvième siècle bourgeois, ne pouvait guère qu'être ineffective, dans la mesure où elle ne se départait jamais vraiment, même dans l'absence et l'obscurité, de la psychologisation et de l'esthétisme vain. Avec Paul Ricœur néanmoins, il est possible de répondre à Sartre que toute résistance compte, précisément puisque nous sommes tous compromis, et ce dès le début et pour toujours.

\section{Abstract}

In our disenchanted world, where no 'universal reader' eagerly expects us to produce anything, and any external validation is necessarily circumscribed and conditional, it can be more and more difficult to convince ourselves that there is any point in writing. Some two centuries ago, Maine de Biran and Samuel Taylor Coleridge realised that the increasingly utilitarian climate meant that the main criterion, when it came to the success of literary production, had become immediate gratification. This is what led them to write, on the side of a number of scattered and sometimes confusing publications, against their readership, in their respective Journal and Notebooks. These writings were de facto a means of resisting against the implacable logic of the need for external recognition, which Biran and Coleridge knew to be, at heart, unquenchable. Jean-Paul Sartre, however, claimed that the struggle of most of nineteenth-century literature against its bourgeois audience was bound to fail, given that it only ever revolved around vain psychological and aesthetic matters. But, as Paul Ricœur suggests, this 
assimilation of literary negation with its opposite rests on a restricted view of both being and nothingness. If we are all in it together, and no one can be deemed fully uncompromised, then any form of resistance, impure as it may be, counts.

\section{Texte de l'article}

Injustifiable comme tous les hommes, l'écrivain envie parfois le privilège de ceux dont l'action semble se justifier immédiatement. ${ }^{1}$

Ecrire sur l'acte de ne pas écrire revient sans doute à rompre le silence pour expliquer pourquoi on le garde ; l'attitude ainsi justifiée s'en trouve du même coup invalidée. Ce genre d'impasse a pourtant le mérite de nous rappeler à l'insolubilité de notre condition, qui veut que les moyens conceptuels dont nous disposons pour faire sens de quelque phénomène que ce soit nous éloignent inévitablement de sa vérité sensible. Tels Orphée perdant Eurydice pour avoir voulu s'assurer qu'elle le suivait, nous dissipons invariablement l'essence de ce que nous n'avons de cesse de saisir. Si cela peut déjà s'avérer problématique lorsqu'il s'agit de déterminer le sens d'un certain nombre de constructions théoriques (telles que république, liberté, égalité, fraternité...) servant de socle au vivre ensemble, la frustration peut s'intensifier lorsqu'il s'agit de se dévoiler ou de se réinventer au travers de mots et de tournures nécessairement gauches, empruntés ou tout simplement impropres. Ainsi que le remarque l'Adolphe de Benjamin Constant, " les sentiments de l'homme sont confus et mélangés; ils se composent d'une multitude d'impressions variées qui échappent à l'observation; et la parole, toujours trop grossière et trop générale, peut bien servir à les désigner, mais ne sert jamais à les définir. ${ }^{2}$

Cette difficulté de se dire entièrement se voit intensifiée par la question du ou des destinataires de nos efforts : écrit-on pour soi, pour les autres, ou pour soi à travers les autres ? Le fond et la forme de nos énoncés dépendent pour une large part de ce que nous cherchons à accomplir, et donc de qui nous cherchons à édifier ou à impressionner. D'après Jean-Paul Sartre, " un des principaux motifs de la création artistique est certainement le besoin de nous sentir essentiels par rapport au monde $\nu^{3}$, et c'est, a priori, « pour le lecteur universel $»^{4}$ que l'on écrit.

Nous pourrions aller plus loin encore et avancer que, dans un monde non encore désenchanté ${ }^{5}$ comme, peut-être, celui d'avant les Lumières et celui des jeunes gens que le cynisme n'a pas encore imprégnés sans retour, la production écrite, comme toute autre forme d'art, correspond à un geste qui transcende les êtres finis pour s'adresser à Dieu, à l'Un ou à l'univers, que l'on soit ouvertement monothéiste, néo-platonicien, panthéiste ou même rien de tout cela. L'idée est simplement que I' " On » nous regarde, et qu'en conséquence tout ce que l'on crée entre en résonnance avec le Tout, pour y laisser une marque indélébile. L'idée, plus simplement encore, est qu'écrire compte, et cela même si personne n'en lit le résultat.

Mais voilà : au sein d'un monde désenchanté comme le nôtre, il est de plus en plus difficile de se convaincre que l'univers nous regarde, et nous sommes alors confrontés à la finitude - la nôtre et celle des autres - et à ses conséquences. L'œuvre ne va plus se fondre dans un absolu qu'elle contribue à ciseler et à colorer ; elle n'est plus guère qu'une production particulière s'adressant à des personnes

\footnotetext{
${ }^{1}$ Claude Roy, Défense de la littérature (Gallimard, Paris, 1968), p.58.

2 Benjamin Constant, Adolphe (Gallimard, Paris, 2007), p. 27.

3 Jean-Paul Sartre, Qu'est-ce que la littérature ? (Paris : Gallimard, 1948), p.46.

${ }^{4}$ Jean-Paul Sartre, Qu'est-ce que la littérature? (Paris : Gallimard, 1948), p.75.

${ }^{5}$ Nous faisons ici référence au terme popularisé par Max Weber, dans L'Ethique protestante et l'esprit du capitalisme (Plon, Paris, 1964).
} 
particulières, présentes ou futures, et dont l'avis ne peut être que relatif. Le papier, le support électronique sont eux-mêmes voués à disparaître, et l'univers n'en a cure.

Sartre situe la naissance du décalage " entre le mythe littéraire et la réalité historique " aux alentours de 1930. C'est à cette époque que les Français (au moins) auraient découvert " avec stupeur leur historicité. ${ }^{6}$ Avant cela, " de la poésie pure à l'écriture automatique, le climat est au platonisme. ${ }^{7}$ Cependant s'il est fort possible que le platonisme, en tant que croyance en une transcendance matrice de sens et de validation, ait présidé jusqu'à il y a peu à l'activité artistique, il est tout autant possible de discerner, bien avant le vingtième siècle, des fêlures dans l'édifice.

Le sentiment $d^{\prime}$ ' à quoi bon " écrire pour un public sinon absent, du moins nécessairement délimité, est ainsi exemplifié par deux penseurs 'mineurs' du tournant du dix-huitième siècle : Maine de Biran (1766-1824) et Samuel Taylor Coleridge (1772-1834). Chez ces deux philosophes qui le deviennent presque par accident, on distingue une réticence à écrire et à publier qui tient non seulement à la peur de ne pas le faire adéquatement, mais aussi et surtout à celle de tomber dans le piège d'une soif de reconnaissance vouée à n'être jamais étanchée.

\section{L’indicible intériorité}

Tout commence avec une rupture épistémologique : tous deux initialement empiristes, dans la lignée de John Locke et de David Hume, au point de concevoir l'esprit humain comme une tabula rasa sur laquelle viendraient s'inscrire les impressions de l'organisme ou des objets externes, Biran et Coleridge se rendent bientôt compte de ce que cette posture prive la nature humaine de tout contrôle sur sa destinée, au point de miner la raison et la liberté si chères à leurs pairs. Biran, disciple d'Etienne Bonnot de Condillac et des Idéologues (au premier rang desquels on trouve Cabanis et Destutt de Tracy), pour lesquels les idées naissent des sensations et constituent un objet d'étude comme un autre, finit par percer l'écran unidimensionnel de leurs catégorisations ${ }^{8}$ en découvrant que l'« effort voulu et immédiatement perçu constitue expressément l'individualité, le moi, ou le fait primitif du sens intime. ${ }^{9}$ Cette localisation de l'étincelle de la conscience de soi dans la confrontation d'une " force hyperorganique " et de la résistance organique ou extérieure remet la volonté à l'honneur, et permet à travers celle-ci de construire l'opposition intériorité/extériorité sur un plan épistémologique, philosophique et psychologique. Coleridge, dont les jeunes années sont marquées par l'influence de l'associationisme de David Hartley et l'enthousiasme pour la Révolution française, laisse finalement là le déterminisme empiriste pour affirmer l'existence, chez l'humain, d'une volonté à la fois voulue par Dieu et indispensable à la conscience de soi et à l'acquisition des connaissances: d'un côté, " l'intelligence et la conscience de soi ne sont possibles que dans et à partir d'une volonté. L'esprit conscient de lui-même est de ce fait une volonté ; et la liberté doit être postulée comme un fondement de la philosophie, et ne peut jamais en être déduite. ${ }^{10}$ De l'autre, « (1) s'il y a en l'Homme quoi que

\footnotetext{
${ }^{6}$ Jean-Paul Sartre, Qu'est-ce que la littérature ? (Paris: Gallimard, 1948), p.212.

7 Jean-Paul Sartre, Qu'est-ce que la littérature ? (Paris : Gallimard, 1948), p.210.

${ }^{8}$ Nous empruntons cette image à Michel Foucault, qui dans Les mots et les choses, déclare que "I'Idéologie n'interroge pas le fondement, les limites ou la racine de la représentation; elle parcourt le domaine des représentations en général ; elle fixe les successions nécessaires qui y apparaissent (...) Elle est en un sens le savoir de tous les savoirs. Mais ce redoublement fondateur ne la fait pas sortir du champ de la représentation ; il a pour fin de rabattre tout savoir sur une représentation à l'immédiateté de laquelle on n'échappe jamais (...) "). En somme, on ne quitte jamais, avec l'Idéologie, "l'espace plat du tableau. " (Michel Foucault, Les mots et les choses, Une archéologie des sciences humaines (Gallimard, Paris, 1966), pp.253-254 \& p.252)

${ }^{9}$ Maine de Biran, Essai sur les fondements de la psychologie (Vrin, Paris, 2001), p.118.

10 'Intelligence or self-consciousness is impossible, except by and in a will. The self-conscious spirit therefore is a will; and freedom must be assumed as a ground for philosophy, and can never be deduced from it.' Samuel Taylor Coleridge, Biographia Literaria (J. M. Dent \& Sons, London, 1975), p.153
} 
ce soit de Spirituel, ce ne peut être que la Volonté. (2) S'il y a une Volonté, il doit y avoir une Spiritualité en l'Homme. " ${ }^{11}$ Là où Biran constate, donc, Coleridge postule.

Dans les deux cas, cela dit, la pensée philosophique suit l'inclination naturelle vers l'exploration d'une intériorité indicible. Mais en opposant ce mystère à un univers philosophique (celui des Lumières et du positivisme naissant) de plus en plus tourné vers l'explicitation et la systématisation, y compris chez les philosophes post-Kantiens, Biran et Coleridge se mettent dans une position ambiguë: si l'intériorité ne peut se communiquer qu'en des termes étrangers à ceux reconnus par la communauté intellectuelle, une philosophie fondée sur cette intériorité se condamne, dans une certaine mesure, à ne s'adresser qu'à elle-même. Cette tension entre le désir impérieux d'exprimer un soi profond qui ne se réduise à aucune forme fixe et un lectorat potentiel en droit de demander de la clarté détermine la forme, voire même l'existence des écrits des deux penseurs, mais aussi de leur rapport à l'écriture luimême.

Biran comme Coleridge éprouvent de grandes difficultés à rendre compte de leurs idées de manière à la fois organisée et fidèle à leurs intuitions. De son vivant, Biran ne publiera que trois travaux, incapable qu'il est de se satisfaire d'une forme spécifique : « je travaille... recommençant et raturant sans cesse... c'est une croix que je me suis donnée... mon ouvrage n'est pas un ami. " ${ }^{12}$ Henri Gouhier observe ainsi que si " l'instinct méditatif ${ }^{13}$ de Biran "trouve une fin dans une œuvre ${ }^{14}$, "cette œuvre introduit une nouvelle contradiction dans sa vie $»^{15}$, puisque « sa vie ne [cesse] de poser des questions qui [remettent] en cause sa philosophie ${ }^{16}$. En somme, "Maine de Biran subit sa vocation. ${ }^{17}$ De son côté, Coleridge est également naturellement réticent à présenter ses idées sous une forme publiable. Ses premiers éclats poétiques, aux côtés de William Wordsworth, pourraient suggérer le contraire ${ }^{18}$, mais le délitement de son mariage, son addiction croissante au laudanum et plus généralement une dépression latente, au début des années 1800, opposent de plus en plus d'obstacles à sa carrière d'homme de lettres. L'état de ses publications reflète cette évolution : de la fondation de périodiques au dessein et à la rédaction obscurs, financés par une souscription bientôt retirée par des lecteurs frustrés, à la parution d'ouvrages philosophico-biographiques tels que la Biographia Literaria (1817), Coleridge n'en finit pas de confondre, voire de décevoir les attentes de son public. On pourrait lui reprocher un manque d'organisation; Humphrey House avance plutôt $q^{\prime} u^{\prime} «$ il est inutile de parler d'une politique d'édition mal définie. II n'y avait aucune politique d'édition. L'idée même de publier angoissait Coleridge au plus haut point. Elle le harcelait et le paralysait. ${ }^{19}$ Coleridge, continue House, tend lorsqu'il écrit à imaginer " un lecteur complètement étranger et plutôt mal disposé à son égard, pas toujours parce qu'il souffre d'un complexe de persécution ou de quelque chose dans ce genre, mais parce qu'il est extrêmement conscient de ce que ce qu'il cherche à exprimer trouve sa source dans une expérience si complexe qu'elle est excessivement difficile à

11 '(1) If there be aught Spiritual in Man, the Will must be such. (2) If there be a Will, there must be a Spirituality in Man.' Samuel Taylor Coleridge, Aids to Reflection, edited by Thomas Fenby (Routledge and Sons, London, 1874), p.91.

12 Maine de Biran, Journal, edité par Henri Gouhier, II (Editions de la Baconnière, Neuchâtel, 1955), p.113.

${ }^{13}$ Henri Gouhier, in Maine de Biran, Oeuvres choisies, édité par Henri Gouhier (Aubier, Paris, 1942), p.16.

${ }^{14}$ Henri Gouhier, in Maine de Biran, Oeuvres choisies, édité par Henri Gouhier (Aubier, Paris 1942), p.16.

${ }^{15}$ Henri Gouhier, in Maine de Biran, Oeuvres choisies, édité par Henri Gouhier (Aubier, Paris, 1942), p.16.

${ }^{16}$ Henri Gouhier, in Maine de Biran, Oeuvres choisies (Aubier, Paris, 1942), p.17.

${ }^{17}$ Henri Gouhier, Maine de Biran: Oeuvres choisies, édité par Henri Gouhier (Aubier, Paris, 1942), p.15.

18 La publication des Lyrical Ballads, en 1798, est l'acte de naissance du romantisme anglais, et consacre Wordsworth et Coleridge comme ses premiers représentants. The Rime of the Ancient Mariner constitue la contribution la plus substantielle de Coleridge à l'ouvrage.

19 'It is useless to speak of a mistaken policy of publication. There was no policy. Coleridge dreaded publication; it harassed him and threw him out.' Humphrey House, Coleridge: The Clark Lectures, 1951-1952 (Rupert HartDavis, London, 1953), p.58. 
présenter et aisément mal comprise. Coleridge était quelqu'un qui sentait que ses expériences les plus précieuses étaient par nature solitaires et que les communiquer aux autres dans une prose engageante et sans aspérités était impossible. $»^{20}$

La frustration de ne pouvoir exprimer leurs idées de manière orthodoxe est doublée, chez les deux penseurs, par la conviction de ce qu'ils sont nés pour accomplir quelque chose, pour mettre au jour une nouvelle vision du monde et de la nature humaine. Biran, nous dit encore Gouhier, "n'a jamais voulu écrire qu'un seul livre, qui eût été un traité de l'homme. ${ }^{21}$ Mais, au vu de l'aspect fragmentaire de sa production, il finit par s'avouer qu'« il est triste de se dire qu'on était né pour faire quelque chose et qu'on n'a rien fait. " $^{22}$ Coleridge, pour sa part, nourrit jusqu'à la fin de sa vie l'espoir d'achever son Opus Maximum, où il compte articuler toutes « ses Pensées autour d'un même principe, dérivé de l'Expérience mais dont tous les autres Savoirs devraient être autant de variations sur un spectre limité, de la même manière que les cercles, les carrés, les triangles etc. ne sont qu'autant de positions dans l'espace. ${ }^{23} \mathrm{~A}$ ce jour, toutes les éditions de l'Opus Maximum demeurent des collections de fragments.

De façon intéressante, donc, la redécouverte et la promotion de la volonté entraîne nos penseurs non pas au dehors, vers l'affirmation de soi dans et sur le monde, mais au-dedans, vers une intériorité inexprimable et faisant obstacle à la première. Le rapport entre volonté et existence sociale se voit en fait, par rapport au discours contemporain sur la question, inversé. Alors que le vouloir est aujourd'hui associé au pouvoir et à la réalisation de projets qui garantissent reconnaissance et appartenance au sein de la société, il est chez Biran et Coleridge le lieu du moi qui s'émancipe de ces désirs instinctuels, et trouve refuge dans une vie intérieure asociale. C'est précisément le désir d'exister socialement, d'être vu, flatté ou applaudi qui signale pour eux l'abdication de la volonté propre.

\section{L’aliénante extériorité}

Cette distinction prend tout son sens si l'on s'avise que Biran et Coleridge, lorsqu'ils affirment la prééminence du vouloir, ne sont pas simplement préoccupés d'épistémologie ; ils cherchent aussi et surtout un moyen de résister aux conséquences morales inévitables du sensualisme de Condillac et de l'associationisme de Hartley. $\mathrm{Si}$, aux termes de ces mouvements de pensée, nos idées sont le produit de nos impressions sensibles, aux termes de l'utilitarisme, qui ne fait que déployer la même logique, nos idées et sentiments moraux ne peuvent qu'être soumis au même régime. "La nature ", dit ainsi le père de l'utilitarisme moderne, Jeremy Bentham, " a placé le genre humain sous le contrôle de deux souverains maîtres : le plaisir et la peine. ${ }^{24}$ Ces deux sensations sont les points cardinaux de la morale utilitariste, et le bonheur y est défini comme la maximisation du premier et l'évitement de la seconde. Peu importe la qualité du ou des plaisirs recherchés; c'est leur intensité et leur nombre qui comptent, pour la simple et bonne raison qu'une action ne peut être jugée bonne ou mauvaise

20 '...an utterly unknown reader whom he imagines to be prejudiced against him, not always because of a persecution feeling or anything like that, but because he is genuinely conscious that what he is trying to say springs from a complexity of experience which is exceedingly difficult to present and may easily be misunderstood.' Humphrey House, Coleridge: The Clark Lectures, 1951-1952 (Rupert Hart-Davis, London, 1953), p.23.

${ }^{21}$ Henri Gouhier, in Maine de Biran, Oeuvres choisies, édité par Henri Gouhier (Paris : Aubier, 1942), p.16.

22 Maine de Biran, Journal, edité par Henri Gouhier, I (Neuchâtel: Editions de la Baconnière, 1954), p.102.

23 ....my Thoughts in some principle, that was derived from Experience, but of which all other Knowledges should be but many repetitions under various limitations, even as circles, squares, triangles, \&c are but so many positions of space.' Samuel Taylor Coleridge, Opus Maximum, edited by Thomas McFarland (Routledge and Kegan Paul, London \& Princeton University Press, Princeton, 2002), xli.

24 'Nature has placed mankind under the governance of two sovereign masters, pain and pleasure.' Jeremy Bentham, Introduction to the Principles of Morals and Legislation (William Pickering, London, 1823), p.1. 
que s'il est possible d'en mesurer l'impact sur le bien-être d'un ou de plusieurs individus, et que la mesure n'admet que ce sur quoi l'on peut « objectivement " s'entendre. Mais c'est précisément là que le bât blesse : si l'on ne s'autorise pas à discuter de l'aspect qualitatif des plaisirs, et si c'est du plaisir brut que dépend la validité d'une action, alors le plaisir procuré par la reconnaissance vaut bien celui procuré par la satisfaction du travail bien fait, même si le travail n'a pas été, en fait, bien fait, ou fait du tout ; alors le plaisir procuré par la gloire vite gagnée vaut, sinon l'emporte sur le plaisir d'avoir accompli quelque chose qui mérite reconnaissance, mais en est dépourvu.

L'une des principales caractéristiques de l'univers libéral-démocratique tel que justifié par Bentham et étudié, plus tard, par le Tocqueville de la Démocratie en Amérique, " est le goût qu'y éprouvent tous les hommes pour les succès faciles et les jouissances présentes. Ceci se retrouve dans les carrières intellectuelles comme dans toutes les autres. "Le succès lui-même se mesure à l'aune de l'approbation par la majorité, qui «se charge de fournir aux individus une foule d'opinions toutes faites, et les soulage ainsi de l'obligation de s'en former qui leur soient propres. » Mais cette quête permanente de validation externe, au lieu de mener à un bonheur serein, n'entraîne souvent qu'inquiétude et agitation. Agnès Antoine voit cela à l'œuvre chez Maine de Biran et ses contemporains, dont la conscience est " malheureuse, parce que sans cesse jugée, et [...] divisée, parce que condamnée à paraître, pour son avantage, autre qu'elle est. ${ }^{25}$ Biran se l'avoue : "j'ai toujours voulu, je veux encore paraître ce que je ne suis pas, et je néglige trop ce que je pourrais être. ${ }^{26}$ Coleridge lui-même fait souvent état de son obsession à la fois pathologique et défiante pour la réputation, " prise dans son sens épistémologique, comme les opinions de ceux qui re-supposent les suppositions des autres ${ }^{27}$, pour affirmer à chaque fois que, cette fois-ci, il s'en est détaché pour de bon. Ce qui ne l'empêche pas, selon William Hazlitt, de régulièrement se complaire dans l'usage de ses talents d'orateur au point de « [poser] sa plume pour s'assurer d'un auditeur, et [d'hypothéquer] l'admiration de la postérité pour l'attention d'un badaud. ${ }^{28}$

Ces inquiétudes de nos deux penseurs quant à l'irrésistibilité grandissante du désir si humain (le leur et celui des autres) d'être reconnu et célébré instantanément peuvent trouver d'autant plus de résonnance dans une société comme la nôtre où, en quelques lignes ou en quelques photos sur les réseaux sociaux, ou en quelques notes fredonnées avec affectation sur un plateau télévisé, on peut s'assurer un public immense et un sentiment de communion et de consécration encore inimaginables il y a de cela vingt ans. Dans ce contexte où tout finit par se rejoindre et se valoir, le poète fébrile aux vers maladroits mais sincères côtoie de près, jusqu'à parfois partager le même espace virtuel, la maxime galvaudée passée d'internaute en internaute, ou les saillies circonstanciées et mal orthographiées de ces derniers. Pire, notre poète se verra sans doute éclipsé par le compte Instagram d'une célébrité vantant les mérites d'une gamme de produits de beauté, ou d'une autre célébrité célèbre pour avoir eu la bonne fortune de l'être. Mais, dira-t-on, n'est-ce pas là l'effet inévitable d'une démocratisation elle-même non seulement inévitable mais aussi souhaitable, en ce qu'elle permet à chacun de trouver son public ? Cette démocratisation, Tocqueville en a cela dit comme nous l'avons vu montré les limites : la liberté individuelle, que ce soit celle de l'artiste ou celle du public, s'y voit contrainte de l'intérieur, par la recherche du plaisir immédiat, et de l'extérieur, par le joug de la majorité. Plus encore, la difficulté dans le contexte présent d'exister aux yeux d'une masse toujours mouvante est de nature à plonger l'individu qui ne sait pas s'y adapter dans une anxiété sourde, sinon un découragement complet. Pourquoi continuer à écrire lorsque ni Dieu, ni le « lecteur universel », ni

\footnotetext{
${ }^{25}$ Agnès Antoine, Maine de Biran. Sujet et politique (Presses Universitaires de France, Paris, 1999), p. 41.

${ }^{26}$ Maine de Biran, Journal, edité par Henri Gouhier, I (Editions de la Baconnière, Neuchâtel, 1954), p.160.

27 '...Reputation, in its etymological sense, as the opinions of those who re-suppose the suppositions of others.' Samuel Taylor Coleridge, Letters, edited by E. L. Griggs, vol. 3 (Clarendon Press, Oxford, 1959), pp.277-278. 28 '...he lays down his pen to make sure of an auditor, and mortgages the admiration of posterity for the stare of an idler.' William Hazlitt, Selected Writings (Penguin Classics, London, 1985), p.233.
} 
même un nombre de gens suffisant ne sont susceptibles de s'intéresser à ce que l'on produit, ou si ce que l'on produit n'a, finalement, pas plus d'importance qu'un commentaire facétieux sur YouTube ? Pourquoi en effet. Si chercher à se faire lire revient à jouer à un jeu que l'on ne peut pas gagner, et auquel on ne peut jouer sans perdre la seule validation sur laquelle on puisse compter, la sienne propre, alors seules deux options demeurent : ne pas écrire du tout, ou n'écrire pour personne d'autre que pour soi, en tournant le dos au public. Dans les deux cas, il y a résistance : résistance au fait d'écrire lui-même, ou résistance au fait de rendre visible ce que l'on écrit.

\section{La contre-écriture}

Biran et Coleridge, qui n'avaient pas à se soucier d'Instagram ni de YouTube, mais qui pouvaient d'ores et déjà ressentir les effets de l'utilitarisme et de la démocratisation du marché de la littérature, ont pu ainsi se réfugier dans une écriture "résistante ", ou " clandestine ", en marge de leur production publique. C'est dans leurs écrits intimes - son Journal pour Biran et ses Notebooks pour Coleridge que prend véritablement corps la tension permanente, chez eux, entre validation intérieure et validation extérieure.

"L'opposition dialectique entre intériorité et extériorité est la substance même du Journal, la pierre de touche de la réflexion biranienne [...] ${ }^{29}$, dit ainsi Agnès Antoine. Dans ses cahiers, Biran évoque sans répit ses états d'âme les moins attractifs: anxiété, désespoir, jalousie, mépris de soi, susceptibilité au climat... Ces notes peuvent servir de fondement à sa philosophie du moi, mais ce n'est pas là leur fonction principale. Henri Gouhier, dans son Introduction, remarque ainsi que « de multiples intentions dictent à Biran les pages que l'on appelle son 'journal intime', sauf, semble-t-il, celle de se raconter pour le public. II pense au public seulement lorsqu'il rassemble les matériaux en vue du livre où ils perdront leur caractère personnel [...]. " Anne Devarieux voit plus précisément le Journal comme l'un des "points d'appui " que Biran n'a de cesse de chercher au milieu de son existence fluide et dispersée, et dont la volonté est le plus proéminent : " en ce sens le Journal est la prière profane de Biran, incantation capable comme la prière 'vraie' d'entretenir, de perpétuer, ce qui est voué à l'instant et à la disparition ou l'occultation. ${ }^{30}$ Le moi biranien $s^{\prime} y$ interprète au plus près de sa source, qui n'est pas encore figée en tel ou tel type d'écrit. Plus encore, cette source y apparaît jaillir de l'effort de s'écrire, dans un mouvement qui rappelle l'effort duquel jaillit le moi conscient. Le constat premier est le plus souvent celui d'un vide ou d'une apathie dont l'existence productrice doit s'extirper : « Toujours mêmes distractions et visites ; au-dedans et au dehors, même incapacité, même vide et nullité d'existence. " $^{31}$ Ou encore : « Je suis plus agité et troublé qu'à l'ordinaire, il n'y a plus aucun aplomb dans mon être. Je ne pense à rien, je ne suis à rien. " ${ }^{32}$ Bientôt s'esquissent la nature du problème, qui a invariablement trait à l'abandon aux sens, et les contours de la solution, qui passe par une reprise en main des facultés actives de l'esprit : " Je me reproche, au fond de ma conscience, de trop songer encore à ces amusements qui nous font passer, sans nous apercevoir, du temps à l'éternité. Je me reproche de ne pas assez approfondir la vie, de n'en pas cultiver assez la partie sérieuse [...] Je ne fais guère que suivre passivement la direction de mon instinct et de mes habitudes. ${ }^{33}$ Le Journal de Biran est ainsi le lieu d'un recueillement et d'un recommencement éternels qui ne peuvent avoir lieu que dans l'absence la plus totale de l'autre.

\footnotetext{
${ }^{29}$ Agnès Antoine, Maine de Biran. Sujet et politique (Presses Universitaires de France, Paris, 1999), p. 44.

${ }^{30}$ Anne Devarieux, Maine de Biran. L’individualité persévérante (Jérôme Millon, Grenoble, 2004), p.264.

${ }^{31}$ Maine de Biran, Journal, edité par Henri Gouhier, III (Neuchâtel: Editions de la Baconnière, 1957), p.64.

32 Maine de Biran, Journal, edité par Henri Gouhier, III (Neuchâtel: Editions de la Baconnière, 1957), p.64.

${ }^{33}$ Maine de Biran, Journal, edité par Henri Gouhier, III (Neuchâtel: Editions de la Baconnière, 1957), p.95.
} 
Coleridge, dans l'une des entrées de ses Notebooks, affirme que « la partie la plus grande, peut-être la plus noble, et certainement la plus subtile de notre nature, doit être solitaire ${ }^{34}$, et si ses carnets sont aussi kaléidoscopiques que le monde auquel il ne peut s'empêcher de réagir (des reflets du soleil sur les truites aux mérites comparés d'Aristote et de Francis Bacon), ils sont aussi l'espace où, enfin, il se retrouve " seul avec lui-même. " $^{35}$ Pierre Pachet va plus loin encore, en se demandant si " prêter intensément et comme excessivement attention au monde dans sa diversité, n'est-ce pas au contraire sournoisement se refuser ? " ${ }^{36}$ Se refuser au repos de l'acceptation des choses telles qu'elles sont données, à leur compartimentation en entités connues et prédéfinies. Coleridge cherche à revenir à la fluidité de l'expérience première, ainsi qu'à l'étonnement originel que celle-ci inspire - une expérience et un étonnement qui précèdent la communication, et peuvent parfois souffrir de son impératif de clarté. Car " le langage et tous les symboles donnent de l'extériorité aux Pensées ${ }^{37}$, et coupent ces dernières de leurs racines. C'est au risque de la solidification qu'il s'agit de parer : " ce que nous rencontrons à l'aube de notre esprit tend à devenir un fétiche, pour la plupart d'entre nous au moins. Bénis soit celui qui voit d'abord l'étoile du matin, sinon le soleil, ou les nuages pourpres, ses hérauts. ${ }^{38}$ Au niveau de l'expérience première, il existe une connivence secrète entre les choses, qui invite à user de l'analogie et du parallèle : « les ombres sur le Blé \& les Bois comme le mouvement de I'Air dans les Voiles. " ${ }^{39} \mathrm{Ou}$ encore: "Brouillard montant en vapeur des profondes fissures ou solutions de continuité des montagnes comme d'un énorme Chaudron - Cascade, que je regarde longuement et de manière soutenue, roulée comme un segment de boue, la roche noire luisant au travers - parmi le vacarme un bruit comme d'innombrables sauterelles ou d'une manufacture de rouets. " ${ }^{40}$ De là des réflexions qui s'évertuent à faire des connexions psychologiques ou métaphysiques entre les phénomènes, à y déceler une certaine continuité : " déduire les instincts d"obscures réminiscences d'un Etat préexistant - j'y ai souvent songé. ${ }^{41} \mathrm{Ou}$ : « en regardant les objets dd la Nature tandis que je réfléchis, comme cette lune là-bas qui luit confusément à travers la vitre emperlée, il semble que je cherche, que je demande en quelque sorte un langage symbolique pour quelque chose en moi qui existe déjà et pour toujours [...]. »" ${ }^{42}$ Ou enfin : " le Cercle tranquille dans lequel le Changement et la Permanence coexistent non par combinaison ou juxtaposition, mais par une annihilation absolue de la différence / colonne de fumée, les fontaines devant St Pierre, les Cascades / DIEU ! - Changement sans perte - changement par une perpétuelle croissance qui <à la

34 '...the greater and, perhaps, nobler, certainly all the subtler, parts of one's nature must be solitary.' Samuel Taylor Coleridge, Coleridge: Select Poetry \& Prose, edited by Stephen Potter (The Nonesuch Press, London, 1933), p.161.

35 Paul Deschamps, La formation de la pensée de Coleridge (1772-1804) (Didier, Paris, 1964), p.25.

${ }^{36}$ Pierre Pachet, in Samuel Taylor Coleridge, Carnets, choix de textes traduits par Pierre Leyris (Belin, Paris, 1987), p.26.

${ }^{37}$ Pierre Pachet, in Samuel Taylor Coleridge, Carnets, choix de textes traduits par Pierre Leyris (Belin, Paris, 1987), p.80.

38 '...that which we first meet with in the dawn of our mind becomes ever after fetish, to the many at least. Blessed he who first sees the morning star, if not the sun, or purpling clouds his harbingers' Samuel Taylor Coleridge, Coleridge: Select Poetry \& Prose, edited by Stephen Potter (The Nonesuch Press, London, 1933), p.180.

${ }^{39}$ Pierre Pachet, in Samuel Taylor Coleridge, Carnets, choix de textes traduits par Pierre Leyris (Belin, Paris, 1987), p.85.

${ }^{40}$ Pierre Pachet, in Samuel Taylor Coleridge, Carnets, choix de textes traduits par Pierre Leyris (Belin, Paris, 1987), p.89.

${ }^{41}$ Pierre Pachet, in Samuel Taylor Coleridge, Carnets, choix de textes traduits par Pierre Leyris (Belin, Paris, 1987), p.155.

42 Pierre Pachet, in Samuel Taylor Coleridge, Carnets, choix de textes traduits par Pierre Leyris (Belin, Paris, 1987), p.170. 
fois constitue et annihile le changement> le passé $\&$ le futur inclus dans le Présent [...]. $"{ }^{43}$ Ces observations tirent comme chez Biran leur préciosité d'un rapport intime et solitaire au monde.

Il s'agit donc, pour Coleridge comme pour Biran, de cultiver une intériorité via un medium qui soit l'extension de cette intériorité. C'est dans cet espace en marge, écrit pour ainsi dire contre l'œuvre publique et le public lui-même, que se tisse ce qui constitue le substrat de l'œuvre elle-même. On peut parler ici, à la manière de Roger Laporte, d'un « affrontement entre [...] l'écriture et la contre-écriture, adversaire qu'il serait pourtant injuste de considérer comme ennemi de l'ouvrage, puisque sans elle l'ouvrage se géométriserait, se cristalliserait en un Traité qui, pour être parfait, n'en signifierait pas moins pour l'ouvrage, sublimé en l'đEuvre, une immobilité mortelle. La contre-écriture, inséparable de l'œuvre, est donc porteuse de vie [...]. " ${ }^{44}$ André Miguel voit dans cet affrontement le lot de la véritable poésie, dont le "refus ouvert " permet "le saut dans le dehors du dedans. [La poésie] prodigue de l'espoir à un espace secret, mobile, fluide, jamais asservi à l'écriture, au texte, au livre. ${ }^{45}$ Le Journal de Biran et les Notebooks de Coleridge sont donc un lieu à la fois fermé et ouvert, et ouvert précisément parce qu'il est fermé à l'espace public, qui tend à cristalliser et donc à réduire le sens toujours évolutif de la pensée et des sentiments et imaginaires qui la nourrissent. Mais cette écriture intime est une écriture intentionnellement dépourvue de lien avec l'autre, et de fait tronquée dans sa pureté même. Le refus d'écrire pour l'autre tend à annuler l'écriture elle-même, si l'on voit l'expression comme indissociable de la communication ${ }^{46}$, et pourtant c'est uniquement dans ce refus qu'elle peut librement se faire. Il y a donc, chez Biran et Coleridge, une résistance à la " littérature ", comprise comme une œuvre organisée qui s'adresserait au « lecteur universel », mais cette résistance est aussi impossible qu'elle est nécessaire. Qui plus est, alors que l'écriture résistante s'insinue surtout contre le fait de devoir exister aux yeux des autres, cette même écriture n'aurait jamais pu venir compléter, voire parachever les œuvres respectives des deux auteurs sans l'intervention des autres, que ceux-ci fussent amis, exécuteurs testamentaires, ou éditeurs. De fait la tension entre intériorité et extériorité demeure inévitable et irrésoluble, et c'est elle qui façonne les écrits intimes de nos deux penseurs, davantage qu'une intériorité pure. Cette tension qui confine à l'interdépendance correspond aux philosophies respectives de Biran et Coleridge, qui encouragent l'individualité mais la définissent de telle façon qu'elle ne peut se construire qu'en rapport avec ce qui n'est pas elle. Chez Biran, c'est la résistance organique ou extérieure qui permet à la force hyperorganique de prendre conscience d'elle-même à travers l'effort; chez Coleridge, la "tendance vers l'individuation progressive " de la vie est inséparable de sa "polarité incessante [...], en tant que forme de son processus. $"{ }^{47}$ La polarité est ainsi condition du dynamisme qui permet l'individuation.

Il est donc impossible de sortir de cette dialectique entre individualité et altérité. L'Autre - le lecteur absent comme le lecteur présent - est un mal nécessaire ; nécessaire parce que la reconnaissance de notre individualité lui revient, et mal précisément pour la même raison. Ce qui reste, malgré tout, c'est le geste ; celui de s'inscrire contre un monde qui dicte les conditions du succès, et impose le succès lui-même comme raison d'être de l'écriture.

\footnotetext{
${ }^{43}$ Pierre Pachet, in Samuel Taylor Coleridge, Carnets, choix de textes traduits par Pierre Leyris (Belin, Paris, 1987), p.183.

${ }^{44}$ Roger Laporte, in André Miguel, L'homme poétique (Editions Saint-Germain-des-Prés, Paris, 1974), p.14.

${ }^{45}$ André Miguel, L'homme poétique (Editions Saint-Germain-des-Prés, Paris, 1974), p.14.

${ }^{46}$ Comme le suggèrent, entre autres, Jean-Paul Sartre et Claude Roy.

47 '...the unceasing polarity of life, as the form of its process, and its tendency to progressive individuation as the law of its direction.' Samuel Taylor Coleridge, Hints toward the Formation of a More Comprehensive Theory of Life (Lea and Blanchard, Philadelphia, 1848), p.67.
} 


\section{L’impossible résistance}

Le problème est que le geste de résistance peut être considéré comme faisant lui-même partie du système qu'il entend contester. II ne serait dans ce contexte qu'un message parmi d'autres, ou pis, une rébellion validée, voire invitée par un système " total ", à la 1984 de George Orwell. Si l'on en croit Sartre, c'est l'écueil dans lequel a donné une bonne partie de la littérature du dix-neuvième siècle, aux prises avec la logique utilitariste de la bourgeoisie triomphante : "les extrémistes souhaitent, par terreur de servir, que leurs ouvrages ne puissent pas même éclairer le lecteur sur son propre cœur, ils refusent de transmettre leur expérience. A la limite l'œuvre ne sera tout à fait gratuite que si elle est tout à fait inhumaine. Au bout de cela, il y a l'espoir d'une création absolue [...], inutilisable en ce monde parce qu'elle $n^{\prime}$ est pas $d u$ monde et qu'elle n'en rappelle rien [...]. » ${ }^{48}$ Mais voilà : "la littérature comme Négation absolue devient l'Anti-littérature ; jamais elle n'a été plus littéraire : la boucle est bouclée. ${ }^{49}$ Ecrire pour les autres et refuser de le faire reviennent au même. Mais il y a plus : I'auteur continue de jouer le jeu de ceux à qui il croit résister : " la bourgeoisie sait bien que l'écrivain a pris secrètement son parti : il a besoin d'elle pour justifier son esthétique d'opposition et de ressentiment [...] il vaut mieux contenir les forces de négation dans un vain esthétisme, dans une révolte sans effet. $"{ }^{50}$ Cette logique Orwellienne d'admet bien entendu aucun échappatoire. Sauf, cela dit, à s'extirper de l'opposition sartrienne entre être et néant. Paul Ricœur remarque ainsi que " la philosophie du néant [de Sartre] est la conséquence d'une philosophie insuffisante de l'être. ${ }^{51}$ L'être sartrien est pour ainsi dire "chosifié ", et c'est ainsi que "toute possibilité de fonder les actes néantisants dans une affirmation supérieure est exclue sous peine de retomber à l'engluement initial ; l'être ne peut plus être recours, mais piège ; glu, mais non élan et fondement [...]. " $^{52} L^{\prime}$ élan, voilà ce que Sartre ne semble pas prendre en compte. N'est-ce pas d'élan et de tension que la résistance est faite ? Résister, cela ne veut pas nécessairement dire être au dehors, ou du moins être complètement sorti du paradigme que l'on entend renverser. Résister, c'est être aux prises avec ce qui pose problème, et le lui faire sentir, quand bien même la lutte serait imparfaite ou inefficace. Le problème de l'approche sartrienne, si l'on peut se permettre d'en parler en ces termes, est au moins double : en premier lieu, réduire une bonne partie de la littérature du dix-neuvième siècle à un repli sur des positions psychologiquement et esthétiquement différentes de celles de la bourgeoisie régnante est non seulement incorrect, c'est aussi méconnaître le lien fait par des penseurs comme Biran et Coleridge eux-mêmes entre l'hégémonie socio-économique et la croyance en un certain nombre de présupposés philosophiques et épistémologiques (seule la gratification immédiate compte, le statut vaut l'effort, etc.) En second lieu, parier sur une littérature " concrète " qui soit à la fois " négativité " et " projet ", " arrachement " et " fête ", et en appelle à la liberté de tous revient à donner dans un certain messianisme, dans la croyance en une certaine pureté de l'écriture, qui disqualifie les tentatives et les intentions de ceux qui, en dépit de leurs efforts, sont donc condamnés à être à jamais englués dans l'être qu'ils essaient vainement de nier. Finalement, Sartre revient au platonisme qu'il s'évertuait à combattre. Le fait est que tout geste compte autant qu'il ne compte pas ; l'écriture est aussi faillible et fragile que ceux qui la font, et elle n'aide que dans la mesure où elle peut aider des êtres finis et conscients de leur finitude.

Futile peut-être, une contre-écriture telle que celle pratiquée par Biran et Coleridge a au moins le mérite de porter à son paroxysme cet « injustifiable » dont Claude Roy fait l'une des principales caractéristiques de l'humanité.

\footnotetext{
48 Jean-Paul Sartre, Qu'est-ce que la littérature ? (Gallimard, Paris, 1948), pp.135-136.

49 Jean-Paul Sartre, Qu'est-ce que la littérature ? (Gallimard, Paris, 1948), p.138.

50 Jean-Paul Sartre, Qu'est-ce que la littérature ? (Gallimard, Paris 1948), pp.140-141.

51 Paul Ricoeur, Anthologie, éditée par Michaël Foessel et Fabien Lamouche (Points, Paris, 2007), p.249.

52 Paul Ricoeur, Anthologie, éditée par Michaël Foessel et Fabien Lamouche (Points, Paris, 2007), p.250.
} 


\section{Ouvrages cités :}

Antoine, Agnès, Maine de Biran. Sujet et politique (Presses Universitaires de France, Paris, 1999)

Bentham, Jeremy, Introduction to the Principles of Morals and Legislation (William Pickering, London, 1823)

Coleridge, Samuel Taylor, Hints toward the Formation of a More Comprehensive Theory of Life (Lea and Blanchard, Philadephia, 1848)

Coleridge, Samuel Taylor, Aids to Reflection, edited by Thomas Fenby (Routledge and Sons, London, 1874)

Coleridge, Samuel Taylor, Coleridge: Select Poetry \& Prose, edited by Stephen Potter (The Nonesuch Press, London, 1933)

Coleridge, Samuel Taylor, Biographia Literaria (London: J. M. Dent \& Sons, 1975)

Coleridge, Samuel Taylor, Carnets, choix de textes traduits par Pierre Leyris (Belin, Paris, 1987)

Coleridge, Samuel Taylor, Opus Maximum, edited by Thomas McFarland (Routledge and Kegan Paul, London \& Princeton University Press, Princeton, 2002)

Constant, Benjamin, Adolphe (Gallimard, Paris, 2007)

Deschamps, Paul, La formation de la pensée de Coleridge (1772-1804) (Didier, Paris, 1964)

Devarieux, Anne, Maine de Biran. L’individualité persévérante (Jérôme Millon, Grenoble, 2004),

Foucault, Michel, Les mots et les choses, Une archéologie des sciences humaines (Gallimard, Paris, 1966)

Hazlitt, William, Selected Writings (Penguin Classics, London, 1985),

House, Humphrey, Coleridge: The Clark Lectures, 1951-1952 (Rupert Hart-Davis, London, 1953)

Maine de Biran, Oeuvres choisies, édité par Henri Gouhier (Aubier, Paris, 1942)

Maine de Biran, Journal, edité par Henri Gouhier, I (Editions de la Baconnière, Neuchâtel, 1954)

Maine de Biran, Journal, edité par Henri Gouhier, II (Editions de la Baconnière, Neuchâtel, 1955)

Maine de Biran, Journal, edité par Henri Gouhier, III (Editions de la Baconnière, Neuchâtel, 1957)

Maine de Biran, Essai sur les fondements de la psychologie (Paris : Vrin, 2001)

Miguel, André, L’homme poétique (Editions Saint-Germain-des-Prés, Paris, 1974)

Ricoeur, Paul, Anthologie, éditée par Michaël Foessel et Fabien Lamouche (Points, Paris, 2007)

Roy, Claude, Défense de la littérature (Gallimard, Paris, 1968)

Sartre, Jean-Paul, Qu'est-ce que la littérature ? (Gallimard, Paris, 1948)

Weber, Max, L'Ethique protestante et l'esprit du capitalisme (Plon, Paris, 1964) 